\title{
Erratum
}

\section{Existence of 3D Skyrmions}

\author{
Maria J. Esteban \\ CEREMADE, Université Paris-Dauphine, 75775 Paris Cedex 16, France
}

Received: 29 October 2003 / Accepted: 14 May 2004

Erratum published online: 3 September 2004 - (C) Springer-Verlag 2004

Commun. Math. Phys. 105, 571-591 (1986)

The Skyrme problem consists in minimizing the energy functional $\mathcal{E}(\phi):=\int_{\mathbb{R}^{3}}|\nabla \phi|^{2}+$ $\left.\sum_{i \neq j} \mid \partial_{i} \phi \wedge \partial_{j} \phi\right)\left.\right|^{2} d x$ in the set of functions $\phi: \mathbb{R}^{3} \rightarrow S^{3}$ such that $\operatorname{deg}(\phi)=$ $\frac{1}{2 \pi^{2}} \int_{\mathbb{R}^{3}} \operatorname{det}(\phi, \nabla \phi) d x=k \in \mathbb{Z}$, the infimum being denoted by $I_{k}$. In $[1,2]$ an existence result for minimizers of degree \pm 1 was proved by using the concentration-compactness method. But as Fanghua Lin and Yisong Yang have pointed out recently [4, 5], the proof of the main result contained in $[1,2]$ is not correct. This Erratum announces that these proofs can be corrected by modifying the arguments used in [1,2]. The method used is still the concentration-compactness principle but applied in a different, and in some sense, less usual way. The new proof, in full detail, has been electronically posted [3].

In a very interesting paper basically devoted to the study of the Faddeev knots ([4])(see also [5] in 2D), F. Lin and Y. Yang have proved recently the existence of $3 D$ Skyrmions of degree \pm 1 by using a different approach, which is based on a cubic decomposition of the whole space. In that paper, they obtain a condition for the existence of solutions for the $3 D$ Skyrme's problem consisting in a family of strict decomposition inequalities. By modifying the proofs in $[1,2]$ but still using the concentration-compactness approach, an existence result for minimizers of $\operatorname{deg}(\phi)= \pm 1$ can be established under the same conditions as in $[4,5]$. This is not surprising. Indeed, the above family of strict inequalities is not only sufficient for the existence of minimizers, but it is in fact necessary and sufficient for the relative compactness of all minimizing sequences. The precise statements of the main results are:

Theorem 1. Let $k \neq 0$. If for all finite integer decompositions of $k, k=\sum_{i=1}^{J} d_{i}$, $d_{i} \in \mathbb{Z}, I_{k}<\sum_{i=1}^{J} I_{d_{i}}$, then, $I_{k}$ is achieved.

Note that in $[1,2]$ only binary decompositions $(J=2)$ had to be avoided. The difference lies in the fact that we do not know anymore whether for all $\ell \in \mathbb{Z} \backslash\{0, k\}$, the large inequalities $I_{k} \leq I_{\ell}+I_{k-\ell}$ hold or not. 
Proposition 1. For all integer $k, 12|k| \pi^{2} \leq I_{k} \leq 12 \sqrt{2}|k| \pi^{2}$.

Corollary 1. The two infima $I_{ \pm 1}$ are achieved, that is, there exist minimizing Skyrmions with degree \pm 1 .

\section{References}

1. Esteban, M.J.: A direct variational approach to Skyrme's model for meson fields. Commun. Math. Phys. 105, 571-591 (1986)

2. Esteban, M.J.: A new setting for Skyrme's Problem. In: Progress in Nonlinear Differential Equations and Their Applications, H. Berestycki, et al. (eds.), Vol. 4, Basel-Boston: Birkhäuser, 1990

3. Esteban, M.J.: Erratum: Existence of $3 D$ Skyrmions. Complete version. http://arxiv.org/abs/math$\mathrm{ph} / 0401042,2004$

4. Lin, F., Yang, Y.: Existence of energy minimizers as stable knotted solitons in the Faddeev model. Commun. Math. Phys. 249, 273-303 (2004)

5. Lin, F., Yang, Y.: Existence of $2 D$ Skyrmions via Concentration-Compactness Method. Preprint

Communicated by M. Aizenman 